\title{
Patients with Parkinson's disease can employ a predictive motor strategy
}

\author{
BL DAY, JPR DICK, CD MARSDEN \\ From the University Department of Neurology, Institute of Psychiatry \& King's College Hospital Medical \\ School, London, UK
}

SUMMARY We have tested the hypothesis that predictive motor behaviour is abnormal in Parkinson's disease. In the first experiment elbow movements were performed to track a moving spot on , an oscilloscope screen. The performance of 12 patients with Parkinson's disease and eight agematched control subjects was measured when tracking a repeated pattern under two conditions. In the first condition subjects were not aware of the repetitive nature of the tracking task whilst in the second condition they were. For both groups tracking error and tracking lag were less when aware of the repetition. In the second experiment wrist movements were studied. Five agematched controls were compared with five patients, studied on and off drugs. In this experiment the performance tracking a repeated pattern was compared to that tracking unpredictable patterns. Tracking lags were reduced to very low values (less than $20 \mathrm{~ms}$ ) in response to the repeated pattern for both groups. This was true even when the patients were relatively immobile off drugs. We conclude that patients with Parkinson's disease are capable of predictive motor behaviour although such a strategy does not always confer as great an advantage in reducing tracking error in patients compared with control subjects.

The functions of the basal ganglia still are uncertain. It is accepted that they are involved in the higher level control of motor performance, but whether they play a more general role in organising other cortical functions, loosely described as cognitive, is debated. Even their role in motor behaviour is controversial. Evidence from human pathology rests heavily on Parkinson's disease. This is not a perfect model of basal ganglia damage in man, for the pathology of Parkinson's disease involves structures other than the nigro-striatal dopamine pathway. Thus, there is involvement of mesocortical dopamine systems, noradrenaline pathways, and even ascending acetylcholine projections to the cerebral cortex in the later stages of the illness. However, Parkinson's disease probably is the best human model of abnormal basal ganglia function available and, in the early stages of the illness, the brunt of pathology falls on the nigro-striatal system.

A striking and consistent motor abnormality in Parkinson's disease, even in its early stages, is slowness in the execution of fast movements.' The Par-

Address for reprint requests: Prof CD Marsden, Institute of
Psychiatry, de Crespigny Park, London SE5 8AF, UK.

Received 20 March 1984. Accepted 29 April 1984 kinsonian patient cannot achieve a normal velocity of fast ballistic movement, but creeps slowly towards the point of aim in a series of small steps. This is because the Parkinsonian cannot inject a large enough initial burst of electromyographic (EMG) activity in the agonist muscle; however, the duration of the first agonist burst is normal, and the timing of subsequent antagonist and agonist bursts is preserved. ${ }^{2}$ The Parkinsonian achieves the movement by a repetitive series of small agonist bursts.

Flowers ${ }^{3}$ pointed out that this failure to produce fast ballistic movement might prevent the Parkinsonian from employing a predictive strategy in movement. When a normal subject undertakes a visual tracking task of an unknown path, he does so. by watching the tracking spot and making appropriate corrective movements after a reaction time. If, however, the tracking spot follows a repetitive predictable path, the subject can switch his strategy to one of moving at the same time, or in advance, of the tracking spot according to a known "internal plan" of the path. ${ }^{4}$ Employment of a predictive strategy is shown by a fall of tracking lag towards zero, and a reduction in tracking error. Flowers suggested that the Parkinsonian's inability to make fast movements would prevent him utilising such a predictive strategy. He went on to show that Parkinsonians 
were unable to improve their tracking error when following a known tracking path, compared with one following a random sequence. ${ }^{5} \mathrm{~A}$ failure to be able to employ a predictive strategy would impose considerable restraints on motor performance in Parkinson's disease. In theory, it would lead to a reliance on external cues and feedback for the execution of all movement which, in turn, would prohibit carrying out any movement faster than the limits imposed by reaction time. The Parkinsonian thus would be constrained to move slowly checking progress at every step.

We have designed experiments to test this hypothesis. A key issue as to whether or not a predictive strategy is employed in a visual tracking task is whether tracking lag can be reduced below reaction time. Whether or not this improves tracking error is a second, but less important, matter. It is quite possible that a subject may employ a predictive strategy, moving in advance of the target, but still not improve greatly on error if each individual movement is constructed incorrectly. We have concentrated, therefore, on whether the Parkinsonian can reduce tracking lag towards zero when presented with a tracking path following a known pattern. Brief preliminary reports of some of this work have been given elsewhere. ${ }^{67}$

\section{Methods}

The patients were requested to perform a tracking task in which they were required to move their elbow (experiment 1) or wrist (experiment 2) to match the movement of a target spot on an oscilloscope screen. The purpose of the experiment was to compare the patients' motor behaviour when tracking a target which moved through either a known or unknown pattern. We have demonstrated previusly that normal subjects change their motor strategy under these two conditions. ${ }^{4}$ Thus, when the target spot moved through a random and unpredictable path, the movement of the subject lagged behind the target movement by approximately one visual reaction time. When the target was then made to repeat its pattern in successive trials, the subject was able to learn the pattern and reduce tracking lag towards zero.

\section{Experiment 1}

Patients with Parkinson's disease, aged 46-79 years (mean $\pm \mathrm{SE}, 67 \pm 3$ years; nine men and three women) were studied. They were selected for their ability to carry out a tracking task despite mild to moderate Parkinsonian disability (Hoehn \& Yahr Grade I-III). The duration of illness varied from $1-26$ years (mean $\pm \mathrm{SE}, 7 \cdot 5 \pm 6 \cdot 8$ years) but none was demented as judged by formal bedside cognitive testing. All exhibited residual Parkinsonian akinesia and rigidity of the right arm, despite drug treatment. Seven had mild tremor. All were taking anti-Parkinsonian drugs at the time of study. These included Sinemet (nine patients), levodopa (two patients), amantadine (four patients) and anticholinergics (three patients). The results obtained in the 12 Parkinsonian patients were compared with those in eight elderly normal subjects aged 52-81 years (mean \pm SE, $67 \pm 3$ years; four males, four females).

The subject was seated with the right arm abducted at the shoulder and with the elbow flexed to $90^{\circ}$. The semipronated forearm was supported on a lever which was pivoted at one end about the centre of rotation of the elbow. This arrangement enabled free flexion and extension of the elbow in a horizontal plane. Subjects grasped a handle which projected at the end of the lever with their right hand. Two $2.5 \mathrm{~cm}$ vertical lines, one beneath the other, were generated on an oscilloscope screen situated one metre in front of the subject at head height. Angular motion of the elbow was transduced by a potentiometer mounted below the manipulandum. The output from the potentiometer was used to control the horizontal position of the bottom line on the oscilloscope screen, $30^{\circ}$ of movement causing $3.53 \mathrm{~cm}$ displacement. Horizontal movement of the top line was controlled by the output from a Hewlett-Packard noise generator (type HOI 3722A).

The subject was instructed to try to keep the arm position spot directly beneath the target position spot at all times. Each trial started with the illumination of a "ready"-light mounted above the screen. One second later the target spot started to move. Movement of the target lasted for a period of $5 \mathrm{~s}$ during which time it moved about its centre position in a random fashion. The frequency spectrum of the target motion was approximately rectangular, with bandwidth $(-3 \mathrm{~dB})$ of $0.5 \mathrm{~Hz}$.

Each subject performed a total of 200 trials. For the first 150 trials the subject was informed that the target movement pattern would be different and unpredictable in each successive trial. In reality, the same target movement pattern was repeated on every third trial, but the complexity of the target pattern was such as to prevent the subject recognising this. In the final 50 trials, the same target pattern was used consecutively. Before starting the final 50 trials, the subjects were informed that the target pattern would now be repetitive.

The arm position and target position signals were converted to digital format, and stored using a PDP 12 computer. The first $1 \mathrm{~s}$ period of each $5 \mathrm{~s}$ trial was not collected. Tracking error was computed over the $4 \mathrm{~s}$ scoring period by subtracting the arm position value from the target position value at each data point, and summing the difference (ignoring the sign). An estimate of average tracking lag was obtained by cross-correlating the arm position trace with the target position trace, and measuring the time delay associated with the maximum value of the crosscorrelation function. The cross-correlation function was obtained using a programme which correlated 256 data points (target position) with a further 256 points (arm position) using a tail-wrapping technique for 64 points on either side of zero shift.

The results of mean tracking error (expressed as a percentage of the error that would have been obtained if the subject had kept his arm stationary-zero movement error), and of mean tracking lag (in ms) were calculated for the first 50 runs of repetitive target movement, interlaced with random trials, during which the subject was unaware 
of the repetitive nature of the task, and for the last 50 runs in which the same repetitive target movement was presented and the subject had been informed that the task was repetitive. The first 10 of each of these 50 trials was discarded, so results are presented for mean tracking error and mean tracking lag for trials $10-50$ in both conditions.

\section{Experiment 2}

In the second experiment we studied five patients with Parkinson's disease both when mobile on effective drug treatment, and immobile when drug therapy had been withdrawn on the day of the experiment. The patients were aged 36-71 years (mean $\pm S E, 59 \pm 15$ years; four males and one female). They had suffered from Parkinson's disease for between $2-20$ years (mean $\pm \mathrm{SE}, 8 \cdot 0 \pm 7 \cdot 2$ years) and were mild to moderately disabled (Hohn \& Yahr Grade I-III). They were taking Sinemet (three patients) or Madopar (two patients), combined with lisuride (one patient), pergolide (one patient) or amantadine (one patient). All were assessed by the Mini-Mental Test and scored 29 points (four patients) or 27 points (one patient), which is well above the limit for detection of cognitive abnormalities (maximum score 30 points). When tested on effective drug treatment, the motor performance of their right arm was indistinguishable from normal. When tested off drug treatment all patients exhibited moderately severe akinesia and rigidity of the right arm, with obvious difficulty in rapid repetitive movement of the limb. For example, when on drug treatment they could rapidly and repetitively execute large movements approximating the ball of the thumb to that of the first finger without difficulty. When off drug treatment, such movements were small, slow, and showed typical Parkinsonian fade. The results obtained in these five patients were compared with those in five normal subjects aged 36-67 years (mean \pm $\mathrm{SE}, 50 \pm 11$ years; two males and three females).

The subjects sat in front of an oscilloscope with their right elbow flexed and forearm semi-pronated resting on a support. The forearm was clamped to the support and the hand, with fingers extended, was held in a splint. This allowed free flexion and extension movements of the wrist in a horizontal plane. Wrist movements were transduced by a Bournes precision potentiometer mounted below and co-axial with the wrist joint. Wrist position was displayed on the oscilloscope as a $1.5 \mathrm{~cm}$ vertical line. Wrist extension moved the line to the right a proportional horizontal distance on the screen $\left(15^{\circ}=1.0 \mathrm{~cm}\right)$. Also displayed on the oscilloscope was a $1.5 \mathrm{~cm}$ horizontal line which represented the target position. When the wrist and target position coincided the two lines formed a cross. The subjects were instructed to try and maintain the cross symmetrical at all times.

Initially, the response to a choice reaction task was measured. A warning light was illuminated above the oscilloscope. One second later, both lines, starting from the top of the oscilloscope screen, moved vertically downwards. When the centre of the screen was reached, the target jumped either to the left or to the right, whilst continuing to move down. The task was to make a rapid and accurate wrist movement so as to re-form the cross. The target line jumped eight times to the left and eight times to the right, in random order, demanding the subject to make eight $20^{\circ}$ extension and eight $20^{\circ}$ flexion movements of the wrist. The time between the start of horizontal movement of the target line, and the subjects' response (taken from when wrist velocity departed from zero) was measured.

Subsequently, the subjects' response to a continuously moving target was studied. The procedure was the same as in the earlier experiment, except that as both lines moved down the screen, the horizontal motion of the target line was controlled by the pseudo-random noise generator, which caused the target line to move continuously in a random fashion for a $4 \mathrm{~s}$ period. The frequency spectrum of the target motion was limited to $(-3 \mathrm{db}) 0.5 \mathrm{~Hz}$. In the first 16 trials, the target line moved through a different pattern in each trial. For the next 24 trials, the target line moved through the same pattern in each trial. Prior to the last 24 trials, the subject was informed that the pattern would be repetitive. At the end of the experiment, each subject was asked to draw on squared paper what they thought had been the path of the target during the repetitive task.

Target position, hand position, hand velocity and a marker signal were recorded on tape (Racal store 7DS FM recorder). This data was converted to digital format ( 250 $\mathrm{Hz}$ sampling frequency) and stored on floppy disc for offline analysis using a PDP 12 computer.

In the initial experiment, reaction time, movement time and total time were measured. Reaction time was defined as the interval between target movement and the earliest detectable hand movement taken from the velocity record. Movement time was defined as the time taken from the start of the hand movement, taken from velocity record, to the point at which the target position was reached and hand velocity fell to zero. Total time was the sum of reaction and movement times.

In the subsequent experiment, the average tracking lag was computed for each trial. However, for the final 24 trials, during which the same target pattern was repeated in consecutive runs, data from the first 16 of these trials were not included in the analysis because of learning effects. Tracking lag was defined as the amount of time, on average, which the subject's hand position lagged behind the target position. Tracking lag was quantified by computing the cross-correlation function between these two variables, as described above. The time shift which yielded the maximum value of the correlation function was taken as an estimate of the average tracking lag (in ms).

In this second experiment, to avoid fatiguing patients, the total number of movements to be performed was kept to a minimum. Therefore, trials which were repeated without the subject's knowledge, as in experiment 1 , were not performed since it required a large number of movements. Repeated trials would need to have been interlaced with ones which were truly unpredictable to avoid detection of repetition by the subject. In addition, this would need to have been accomplished twice since patients were studied on and off drugs. Accordingly, it was impossible to directly compare the Parkinsonians' performance tracking a repeated pattern of which they were unaware, with their tracking ability once they knew the same pattern was repeated. In this situation, therefore, performance had to be compared between tracking a random target pattern with that obtained when tracking a repeated pattern. In these cir- 
Table 1 Normalised tracking error (\%) and tracking lag (ms) from experiment 1

\begin{tabular}{|c|c|c|c|c|c|c|c|c|}
\hline \multirow[t]{2}{*}{ Subject } & \multirow[t]{2}{*}{ Age } & \multirow[t]{2}{*}{ Sex } & \multicolumn{2}{|l|}{$M e a n \pm S E$ error } & \multirow[t]{2}{*}{$p$} & \multicolumn{2}{|l|}{ Mean $\pm S E$ lag } & \multirow[t]{2}{*}{$p$} \\
\hline & & & $\begin{array}{l}\text { Target not known to } \\
\text { be predictable }\end{array}$ & $\begin{array}{l}\text { Target known to be } \\
\text { predictable }\end{array}$ & & $\begin{array}{l}\text { Target not known to } \\
\text { be predictable }\end{array}$ & $\begin{array}{l}\text { Target known to be } \\
\text { predictable }\end{array}$ & \\
\hline $\begin{array}{l}\text { Patients } \\
\text { RM } \\
\text { FC } \\
\text { WD } \\
\text { LB } \\
\text { HF } \\
\text { WL } \\
\text { GR } \\
\text { JM } \\
\text { IH } \\
\text { WG } \\
\text { MM } \\
\text { JE } \\
\text { Mean } \pm \\
\text { Median } \\
\text { Normals } \\
\text { HC } \\
\text { HZ } \\
\text { FW } \\
\text { MF } \\
\text { WH } \\
\text { CS } \\
\text { JL } \\
\text { AM } \\
\text { Mean } \pm \\
\text { Median }\end{array}$ & $\begin{array}{l}74 \\
74 \\
70 \\
74 \\
66 \\
64 \\
79 \\
46 \\
69 \\
64 \\
56 \\
67 \\
E 67 \pm 3 \\
68 \\
\\
69 \\
71 \\
81 \\
68 \\
52 \\
63 \\
66 \\
69 \\
E 67 \pm 3 \\
69\end{array}$ & $\begin{array}{l}\mathrm{F} \\
\mathbf{M} \\
\mathrm{M} \\
\mathrm{M} \\
\mathrm{M} \\
\mathrm{M} \\
\mathrm{M} \\
\mathrm{M} \\
\mathrm{F} \\
\mathrm{M} \\
\mathrm{F} \\
\mathbf{M}\end{array}$ & $\begin{array}{l}69 \pm 1 \\
49 \pm 1 \\
41 \pm 1 \\
53 \pm 1 \\
65 \pm 1 \\
57 \pm 1 \\
64 \pm 1 \\
47 \pm 1 \\
51 \pm 2 \\
50 \pm 1 \\
46 \pm 1 \\
69 \pm 2 \\
55 \pm 3 \\
52 \\
69 \pm 1 \\
46 \pm 1 \\
70 \pm 2 \\
39 \pm 1 \\
41 \pm 1 \\
50 \pm 1 \\
65 \pm 1 \\
46 \pm 1 \\
53 \pm 5 \\
48\end{array}$ & $\begin{array}{l}50 \pm 5 \\
52 \pm 2 \\
41 \pm 1 \\
45 \pm 2 \\
41 \pm 1 \\
54 \pm 1 \\
62 \pm 1 \\
43 \pm 1 \\
31 \pm 1 \\
45 \pm 1 \\
32 \pm 1 \\
81 \pm 2 \\
48 \pm 4 \\
45 \\
35 \pm 1 \\
28 \pm 1 \\
72 \pm 2 \\
34 \pm 1 \\
24 \pm 1 \\
30 \pm 1 \\
31 \pm 1 \\
37 \pm 1 \\
36 \pm 5 \\
33\end{array}$ & $\begin{array}{l}0.001 \\
\text { NS } \\
\text { NS } \\
0.001 \\
0.001 \\
0.05 \\
N S \\
0.001 \\
0.001 \\
0.01 \\
0.001 \\
0.001 \\
0.05\end{array}$ & $\begin{aligned} 143 & \pm 11 \\
52 & \pm 7 \\
69 & \pm 5 \\
59 & \pm 7 \\
118 & \pm 11 \\
158 & \pm 7 \\
144 & \pm 7 \\
86 & \pm 4 \\
111 & \pm 13 \\
83 & \pm 6 \\
86 & \pm 6 \\
191 & \pm 11 \\
108 & \pm 13 \\
99 & \\
190 & \pm 10 \\
92 & \pm 4 \\
178 & \pm 11 \\
73 & \pm 4 \\
54 & \pm 5 \\
115 & \pm 4 \\
222 & \pm 14 \\
100 & \pm 4 \\
128 & \pm 22 \\
108 & \end{aligned}$ & $\begin{aligned} 131 & \pm 7 \\
-33 & \pm 10 \\
49 & \pm 5 \\
18 & \pm 2 \\
57 & \pm 6 \\
122 & \pm 6 \\
65 & \pm 9 \\
65 & \pm 5 \\
21 & \pm 2 \\
40 & \pm 7 \\
26 & \pm 4 \\
127 & \pm 19 \\
57 & \pm 14 \\
53 & \\
& \\
-11 & \pm 6 \\
23 & \pm 4 \\
110 & \pm 16 \\
22 & \pm 2 \\
16 & \pm 1 \\
20 & \pm 2 \\
19 & \pm 4 \\
34 & \pm 1 \\
29 & \pm 12 \\
21 & \end{aligned}$ & $\begin{array}{l}\text { NS } \\
0 \cdot 001 \\
0 \cdot 01 \\
0 \cdot 001 \\
0 \cdot 001 \\
0 \cdot 001 \\
0 \cdot 001 \\
0 \cdot 01 \\
0 \cdot 001 \\
0 \cdot 001 \\
0 \cdot 001 \\
0 \cdot 01 \\
0 \cdot 001\end{array}$ \\
\hline
\end{tabular}

Footnote: Values were obtained from trials 11 to 50 when tracking the same repeated target pattern under two conditions. First, when each repeated target pattern was separated by two trials which were truly random (target not known to be predictable). Second, when each repeated target pattern was presented in consecutive trials with the subject's knowledge (target known to be predictable). Comparison of mean values for each individual were performed using the Student's $t$ test, and that of group data using the paired Student's $t$ test on individual mean values.

cumstances, comparison of tracking lag in the two situations is valid, but comparison of error scores may not be valid since the paths followed by the target in the two situations (random versus repeated paths) were very different. Accordingly, we have concentrated on data on tracking lag only in this experiment.

Statistical analysis was by Student's $t$ test of paired or unpaired data, after comparison of means and medians to establish normal distribution of the data.

\section{Results}

\section{Experiment 1}

The mean error and lag for the repeated trials during the initial phase of the experiment (runs 1-150) when the subjects were unaware that every third run was repetitive, and during the last phase of the experiment (runs 151-200) when the subjects had been told that the task was now repetitive are summarised for the 12 patients with Parkinson's disease and eight control subjects in table 1 .

Mean lags did not differ between patients and controls when they were unaware that the pattern was repetitive ( $\mathrm{p}>0 \cdot \overline{0} \overline{5}$, Student's $t$ test). However, it is notable that individual values for most subjects were below visual reaction time, which in separate similar experiments ${ }^{4}$ averaged some $187 \mathrm{~ms}$. This indicates that many of the subjects were tending to move in advance of the tracking pattern on occasions. This is likely to be a reflection of the slowness of the tracking task, the speed of which was imposed by the necessity to provide a tracking pattern slow enough for patients with Parkinson's disease to follow. In these circumstances, subjects tended to react to a change in direction of the tracking line, after an appropriate lag, by an excessively fast movement to catch and even overtake the pattern. Inevitably, this means a reduction of tracking lag below true visual reaction time. This is analogous to the perceptual anticipation described by Poulton. ${ }^{8}$

When the subjects were told that the pattern was repetitive, tracking lag fell in all control subjects and in 11 of the 12 patients with Parkinson's disease. Mean lag fell from $128 \mathrm{~ms}$ to $29 \mathrm{~ms}(\mathrm{p}<0.001$, paired Student's $t$ test) in control subjects. Mean lag fell from $108 \mathrm{~ms}$ to $57 \mathrm{~ms}(\mathrm{p}<0.001)$ in patients with Parkinson's disease.

Tracking error also improved when the control subjects or the patients realised that the tracking pattern was repetitive. Compared with tracking error during the earlier phase of the experiment, tracking error fell significantly $(p<0.05$ or less, paired Student's $t$ test) in seven of the eight control 


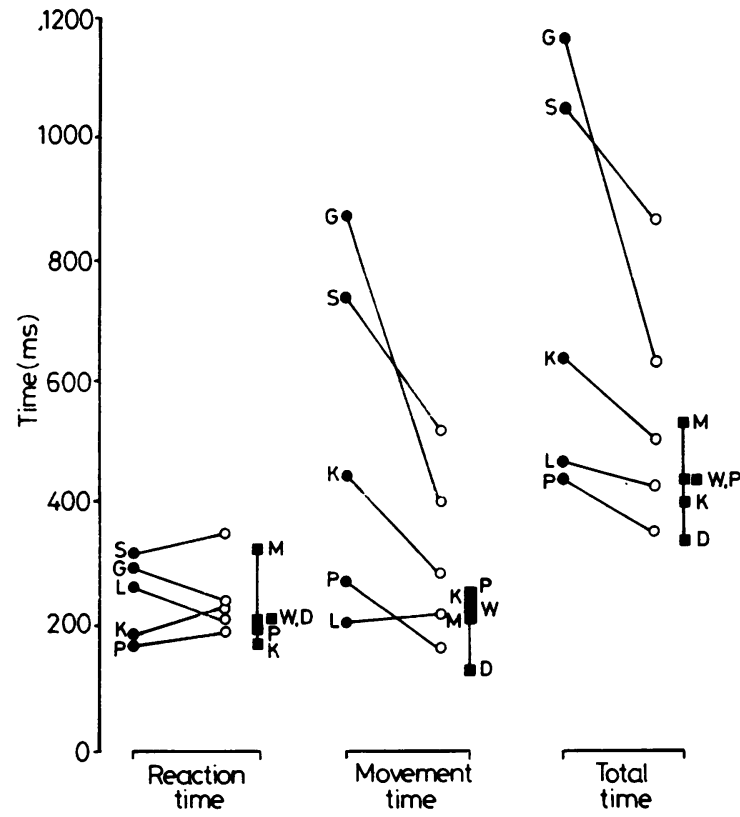

Fig 1 Average reaction time, movement time and total time for eight $20^{\circ}$ wrist flexion and eight $20^{\circ}$ wrist extension movements during a choice reaction task. Reaction time defined as interval between target movement and wrist movement; movement time is interval between initiation of movement and target aquisition; total time is the sum of reaction time and movement time. Solid squares (to the right of each panel) from age-matched control subjects, solid circles from patients with Parkinson's disease OFF drugs and open circles from same patients $O N$ drugs. Individual patients are identified by their last initial.

subjects, and in eight of 12 patients with Parkinson's disease. Mean $( \pm S E)$ tracking error of the unpredictable target was not different in control subjects compared to Parkinsonian patients $(53 \pm 5$ v. $55 \pm$ $3, p>0.05$, Student' $s t$ test), but was less in controls than patients for the predictable target $(36 \pm 5 \mathrm{v} .48$ \pm 4 ) although this difference did not reach statistical significance $(p=0 \cdot 08)$. There was a significant positive correlation between the improvement in tracking error and reduction in tracking lag in control subjects $(r=0.83, p<0.05)$, but not in patients with Parkinson's disease $(r=-0.03, p>0.05)$.

\section{Experiment 2}

The data on reaction time, movement time and total time for the control subjects and the five patients with Parkinson's disease when mobile on drugs and immobile off drugs are summarised in fig 1 . As in other studies ${ }^{9}$ movement time was the best index of Parkinsonian disability and improved in all but one of the five patients when mobile. Total time improved in all five subjects.

Mean tracking lags for control subjects and patients with Parkinson's disease when mobile on drugs and immobile off drugs for both the random patterns and the repetitive patterns are summarised in fig 2. Standard errors of the means for tracking lags were less than $20 \mathrm{~ms}$ for random trials and less than $6 \mathrm{~ms}$ for repeated trials. When the target pattern was random, tracking lags again were less than those expected on the basis of the simple visual reaction time. However, again, the speed of the pattern was deliberately designed to be slow enough for patients with Parkinson's disease to follow, and perceptual anticipation probably was responsible (see above). When the tracking pattern became repetitive, and the subjects were informed of this, tracking lag fell both in all controls and in all patients with Parkinson's disease, whether they were mobile or immobile. Mean ( $\pm \mathrm{SE})$ tracking lag in $\mathrm{ms}$ in controls for random versus predictable target was $110 \pm$ 9 v. $13 \pm 1(p<0.01)$. For Parkinsonians on treatment the equivalent values were $114 \pm 13$ v. $25 \pm$ $12(\mathrm{p}<0.01)$, and off treatment $162 \pm 26 \mathrm{v} .16 \pm 2$ (p $<0.01)$ (paired Student's $t$ test). In fact, mean tracking lag when the pattern was repetitive was no different in normal subjects compared to patients with Parkinson's disease immobile off drugs $(p>$

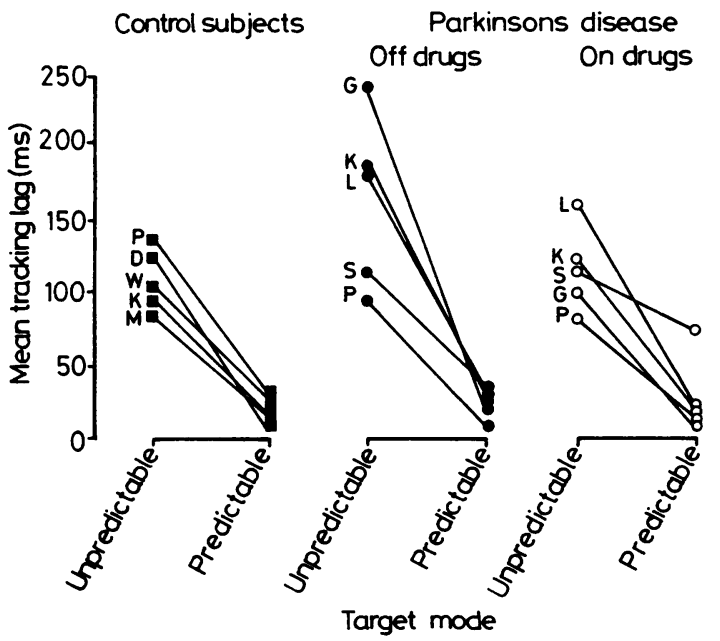

Fig 2 Average tracking lag data in experiment 2 taken from 16 trials when target was unpredictable and eight trials when target was predictable. Solid squares from age-matched control subjects (left panel), solid circles from patients with Parkinson's disease OFF drugs (middle panel) and open circles from same patients $O N$ drugs (right panel). Individual patients are identified by their last initial. 
Table 2 Mean \pm tracking error (\%) from experiment 2

\begin{tabular}{llll}
\hline & \multicolumn{3}{c}{ Subject Target unpredictable Target predictable } \\
\hline Age-matched & & & \\
controls & EW & $37 \pm 4$ & $32 \pm 1$ \\
& PP & $43 \pm 3$ & $37 \pm 2$ \\
& EK & $41 \pm 3$ & $27 \pm 2^{*}$ \\
& SM & $34 \pm 3$ & $38 \pm 3$ \\
& PD & $45 \pm 3$ & $41 \pm 1$ \\
Patients on drugg VK & $40 \pm 2$ & $35 \pm 2$ \\
& TP & $33 \pm 2$ & $35 \pm 2$ \\
& EG & $51 \pm 5$ & $33 \pm 1$ \\
& SL & $54 \pm 3$ & $33 \pm 2^{*}$ \\
& DS & $48 \pm 5$ & $48 \pm 3$ \\
Patients off drugs VK & $45 \pm 4$ & $67 \pm 4^{*}$ \\
& TP & $41 \pm 2$ & $43 \pm 7$ \\
& EG & $60 \pm 3$ & $51 \pm 2$ \\
& SL & $52 \pm 3$ & $42 \pm 2$ \\
& DS & $61 \pm 4$ & $54 \pm 3$ \\
& & $52 \pm 4$ & $40 \pm 3$ \\
\end{tabular}

Values were obtained under two conditions. First, 16 trials during which consecutive target patterns always were different (target unpredictable). Second, the final 8 of 24 trials during which consecutive target patterns were the same (target predictable). Comparison, between predictable and unpredictable conditions, of mean values for each individual were performed using the Student's $t$-test, and that of group data using the paired Student's $t$ test on individual mean values. All comparisons were non-significant except those marked with an asterisk which were significant at the $5 \%$ level.

$0 \cdot 05)$, and the latters' performance was slightly but insignificantly $(p>0 \cdot 05)$ better when they were off drugs compared to on treatment (one patient only reduced tracking lag to about $72 \mathrm{~ms}$, although all the others reduced tracking lag to below $20 \mathrm{~ms}$ whether on or off drugs-fig. 2). Mean tracking lags when the subjects knew the pattern was repetitive fell well within the range of those expected for predictive motor behaviour. ${ }^{4}$

As discussed in Methods, the data on tracking error in this experiment is less reliable, but is presented for completion in table 2 . It is notable that mean tracking error did not improve $(p>0.05$, paired Student's $t$ test) in either control subjects or patients with Parkinson's disease when the target path changed from random to predictable. However, the two tracking target paths were not comparable. Comparison of mean tracking error for the Parkinsonian patients when the target was random showed improvement when on compared to off drugs $(45 \pm 4$ v. $52 \pm 4, p<0.05$, paired Student's $t$ test), and when the target was predictable ( $43 \pm 7$ on v. $50 \pm 4$ off), although this difference did not reach statistical significance $(p>0.05)$.

When the patients were asked to draw the predictable target pattern from memory at the end of the experiment, four of the five were capable of doing so with some accuracy, whether on or off drugs (fig. 3).

\section{Discussion}

The results demonstrate that patients with Parkinson's disease are capable of using a predictive motor strategy when tracking by arm movement a spot on an oscilloscope screen. This was true both for elbow and wrist movements. Despite the obvious motor impairment of these patients their tracking perfor-

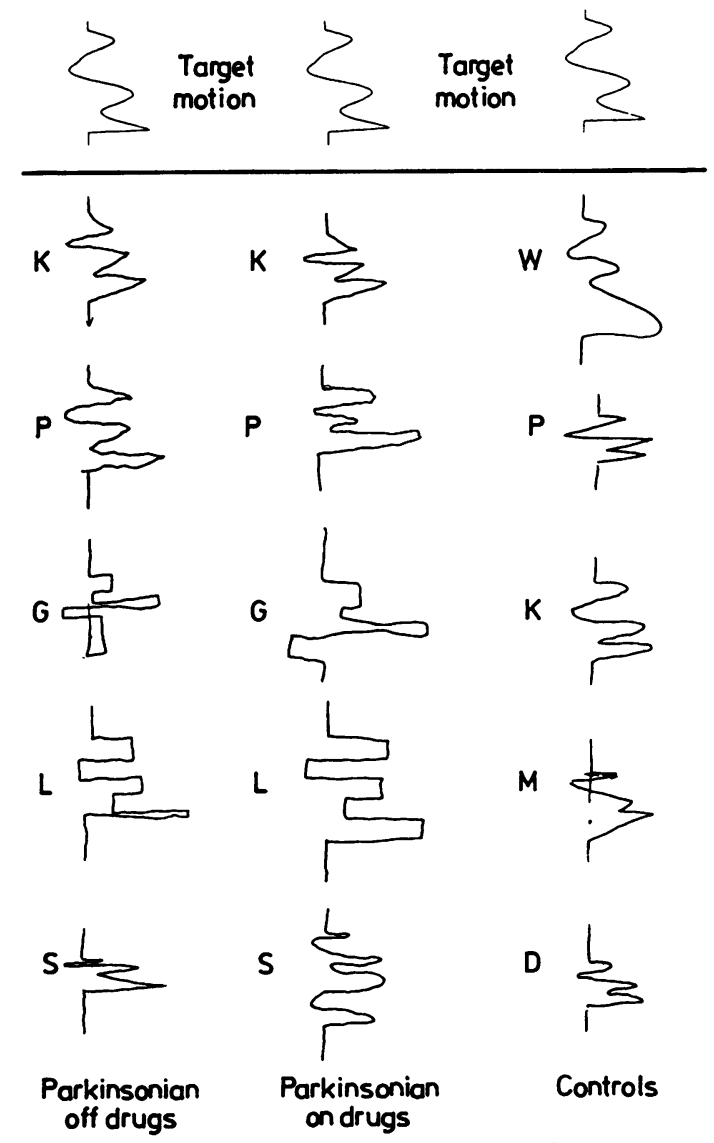

Fig 3 Drawings of target pattern from memory at end of experiment. Top trace in each column is the actual target motion. Left column from patients with Parkinson's disease $O F F$ drugs. Centre column from same patients $O N$ drugs. Right column from age-matched control subjects.

Individual patients are identified by their last initial. Some patients $(G$ and $L$ ) chose to reproduce the pattern in squared format. All the control subjects reproduced the main features of the target pattern, albeit crudely. Of the patients, $K P$ and $L$ also achieved reasonable replicas, whether on or off drugs. $G$ made the same errors of proportion and omission whether on or off drugs. Patient $S$ made a reasonable replica when off drugs, but introduced an extra deviation when on drugs. 
mance, reflected in measurements of tracking lag, was comparable to that of the normal age-matched control subjects. These findings, therefore, do not support the hypothesis that Parkinson's disease may prevent predictive control of arm movements to an extent that allows movement only in response to external stimuli.

When tracking a moving spot, the path of which is unknown, the most successful strategy is to compare current arm position with target position and eliminate the error with a series of corrective arm movements. The frequency of corrective movements depends upon the time required to assess the error, to compute the appropriate motor programme and to execute the corrective movement. Craik ${ }^{10}$ observed that such intermittent corrective movements may be generated at a maximum rate of about $2 / \mathrm{s}$. An unfortunate corollary of this strategy is that movement will lag behind the target with a minimum lag determined by visual reaction time. However, the strategy may be refined by using short-term prediction. Rather than executing corrective movements based upon current target position, the corrective movement may be based upon the estimated target position at a time when the corrective movement is completed. This refinement may help to reduce tracking lag below visual reaction time. Scope for using short-term motor prediction depends upon two factors. Velocity of the target and statistical properties of the target path. ${ }^{8}$ Thus, a slowly moving target with well-defined movement limitations would be more amenable to short-term prediction. In the experiments reported here, patients with Parkinson's disease demonstrated that they were capable of using short-term motor prediction since mean tracking lag, in response to a random target motion or a repeated target motion of which they were unaware, often was below visual reaction time.

When tracking a completely predictable target motion, tracking lag may be reduced further. To achieve this a different motor strategy needs to be implemented. The target pattern is now learnt and motor commands are issued according to an "internal plan" of the target motion rather than in direct response to the actual target motion. Reaction time delays can be circumvented and tracking lags reduced to very low values. In the first experiment the tracking lags of the patients' elbow movements were reduced further when they became aware of the repetitive nature of the tracking task. Similarly, in the second experiment, in which tracking was accomplished by movements of the wrist, the tracking lags were much lower when tracking the repeated pattern compared to the target pattern which changed in successive trials. The fact that patients were able to learn the target pattern and move accordingly was demonstrated further when they reproduced the pattern accurately with pencil and paper after the experiment. Furthermore, the results showed that predictive motor behaviour was uninfluenced by medication.

We conclude that patients with Parkinson's disease are capable of learning a target motion and moving according to an "internal plan" in a predictive fashion. However, the success of such a predictive strategy in reducing tracking error depends upon the accuracy of the movements themselves. Patients with Parkinson's disease are not capable of generating fast accurate movements so, inevitably, tracking error may not improve as much as in control subjects, despite adoption of a predictive strategy. This was evident in both experiments in that patients with Parkinson's disease, despite adopting a predictive strategy once they knew they were tracking a repetitive target, reduced tracking error less than control subjects (experiment 1), or less when off drugs than when on drugs (experiment 2). So the conclusions must be refined as follows: (1) Parkinson's disease does not prevent adoption of predictive motor action or the learning of an internal motor plan, (2) In the present experiments studying simple visual tracking by one joint arm movement, patients with Parkinson's disease automatically chose to adopt such a predictive strategy, (3) However, because their disease prevents accurate fast movement, this does not achieve the gains in accuracy that normal subjects might obtain by such a means of switching motor control, (4) So, for more complex movements, patients with Parkinson's disease might still forego the benefits of predictive action as far as speed is concerned, in order to obtain the accuracy of slower corrective movement.

This work was supported by the Medical Research Council and the Parkinson's Disease Society.

\section{References}

' Wilson SAK. Disorders of motility and muscle tone, with special reference to the striatum. Lancet 1925;2:1-53, 169, 215, 268.

${ }^{2}$ Hallett M, Koshbin S. A physiological mechanism of bradykinesia. Brain 1980;103:301-14.

"Flowers KA. Visual "closed-loop" and "open-loop" characteristics of voluntary movement in patients with Parkinsonism and intention tremor. Brain 1976;99:269-310.

${ }^{4}$ Day BL, Marsden CD. Two strategies for learning a visually guided motor task. Percept Mot. Skills 1983; 55: 1003-16.

${ }^{5}$ Flowers KA. Some frequency response characteristics of Parkinsonism on pursuit tracking. Brain 
1978;101:19-34.

${ }^{6}$ Marsden CD. The mysterious motor function of the basal ganglia: The Robert Wartenberg Lecture. Neurology (NY) 1982:32:514-39.

${ }^{7}$ Marsden CD. Function of the basal ganglia as revealed by cognitive and motor disorders in Parkinson's disease. Can. J Neurol Sci 1984;11:129-35.
${ }^{8}$ Poulton EC. The basis of perceptual anticipation in tracking. Br J Psychol 1952;43:295-302.

${ }^{9}$ Evarts EV, Teravainen H, Calne DB. Reaction time in Parkinson's disease. Brain 1981;104:167-86.

1" Craik KJW. Theory of the human operator in control systems: I, The operator as an engineering system. $\mathrm{Br}$ J Psychol 1947;38:56-61. 